\title{
Production and calibration of the first HEFT hard $x$-ray optics module
}

Jason E. Koglin, C. M. Hubert Chen, James Chonko, Finn E. Christensen, William W. Craig, et al.

Jason E. Koglin, C. M. Hubert Chen, James Chonko, Finn E. Christensen, William W. Craig, Todd R. Decker, Kurt S. Gunderson, Charles J. Hailey, Fiona A. Harrison, Carsten P. Jensen, Kristin K. Madsen, Marcela Stern, David L. Windt, Haitao Yu, Eric Ziegler, "Production and calibration of the first HEFT hard x-ray optics module," Proc. SPIE 5168, Optics for EUV, XRay, and Gamma-Ray Astronomy, (29 January 2004); doi: 10.1117/12.506431 


\title{
Production and calibration of the first HEFT hard X-ray optics module
}

\author{
Jason E. Koglin ${ }^{a^{*}}$, C. M. Hubert Chen ${ }^{\mathrm{b}}$, Jim Chonko ${ }^{\mathrm{a}}$, Finn E. Christensen ${ }^{\mathrm{c}}$, William W. Craig ${ }^{\mathrm{d}}$, \\ Todd R. Decker ${ }^{d}$, Kurt S. Gunderson ${ }^{d}$, Charles J. Hailey ${ }^{a}$, Fiona A. Harrison ${ }^{b}$, Carsten P. Jensen ${ }^{c}$, \\ Kristin K. Madsen ${ }^{c}$, Marcela Stern ${ }^{\mathrm{a}}$, David L. Windt ${ }^{\mathrm{a}}$, Haitao Yu $\mathrm{u}^{\mathrm{a}}$, Eric Ziegler \\ ${ }^{a}$ Columbia Astrophysics Laboratory, New York, NY \\ ${ }^{\mathrm{b}}$ California Institute of Technology, Pasadena, CA \\ ${ }^{c}$ Danish Space Research Institute, Copenhagen, Denmark \\ ${ }^{\mathrm{d}}$ Lawrence Livermore National Laboratory, Livermore, CA \\ ${ }^{\mathrm{e}}$ European Sychrotron Radiation Facility, Grenoble Cedex, France
}

\begin{abstract}
Complete hard X-ray optics modules are currently being produced for the High Energy Focusing Telescope (HEFT), a balloon born mission that will observe a wide range of objects including young supernova remnants, active galactic nuclei, and galaxy clusters at energies between 20 and $70 \mathrm{keV}$. Large collecting areas are achieved by tightly nesting layers of grazing incidence mirrors in a conic approximation Wolter-I design. The segmented layers are made of thermally-formed glass substrates coated with depth-graded multilayer films for enhanced reflectivity. Our novel mounting technique involves constraining these mirror segments to successive layers of precisely machined graphite spacers. We report the production and calibration of the first HEFT optics module.
\end{abstract}

KEYWORDS: Hard X-ray telescope, X-ray optics, segmented glass, thermally-slumped

\section{MOTIVATION FOR HARD X-RAY OPTICS DEVELOPMENT}

Focusing optics for hard X-rays are now poised to open an entirely new window on the high-energy universe. Hard Xray observatories will provide a vehicle for discovering collapsed stars and black holes on all scales, for testing theories of where the elements are born, and for exploring the most extreme environments in the Universe. We have developed thermally-formed glass substrates and a unique mounting technique to build the high performance, lightweight telescopes with large effective area that are necessary to usher in this new era of discovery.

Our approach is currently being demonstrated through the High Energy Focusing Telescope (HEFT), a balloon born mission that is scheduled to fly by Spring 2004. We have already assembled two complete optic modules and more optic modules are already in the process of being assembled for this first flight. Each of these conical-approximation Wolter I optics, comprised of 72 layers of segmented mirrors, will focus onto individual CdZnTe detectors. ${ }^{1}$ Depth-graded W/Si multilayer coatings are used to providing good energy response extending to $70 \mathrm{keV}$. $^{2}$ Future telescopes will use other multilayer materials to increase the response to $100 \mathrm{keV} .^{3}$ Up to fourteen modules can be mounted on HEFT's precision pointing platform which will provide one arcminute pointing stability with 15 " aspect reconstruction. ${ }^{4}$ As a focusing experiment, HEFT crosses two important observational thresholds not approached by previous hard X-ray/soft gammaray instruments: (1) long observations $\left(\sim 2 \times 10^{4} \mathrm{~s}\right.$ for a balloon) that are signal-limited, with sensitivity superior to that achieved by hard X-ray satellite observations, and (2) due to the high sensitivity to faint sources, significant extragalactic astrophysics is possible from a balloon for the first time. In a 6-hour exposure, HEFT reaches a $40 \mathrm{keV}$ flux threshold more than an order of magnitude lower than HEXTE in $10^{5} \mathrm{~s}$. For many science objectives, a single balloon flight is comparable in observation time to an entire satellite mission. In addition, HEFT will provide high spectral resolution at interesting sensitivity levels for the first time ever in this band. Some of the primary scientific objectives for HEFT include imaging and spectroscopy of ${ }^{44} \mathrm{Ti}$ emission in young supernova remnants, sensitive observations of obscured

\footnotetext{
* Correspondence: koglin@astro.columbia.edu, http://www.astro.columbia.edu/ koglin
} 
Active Galactic Nuclei, spectroscopic observations of accreting pulsars with high-magnetic fields, and mapping the Galactic Center.

HEFT will not only be among the first focusing telescopes to yield significant science returns as a balloon mission, it also promises to demonstrate key technologies that are proposed for future satellite missions. Our approach to thermally-forming glass substrates, which is discussed in Section 2, has been adapted to include an epoxy replication step - this epoxy replicated glass has been adopted as the primary fabrication approach for the Constellation-X $($ Con- $X)$ Soft X-ray Telescope (SXT) and is one of two alternatives being considered for the Con-X Hard X-ray Telescope (HXT). Both of these missions, along with NASA's High-Resolution Spectroscopic Imager (HSI) are also considering adopting the HEFT optics mounting approach, which is discussed in Section 3. Together, thermally-formed glass and our mounting approach are being proposed for a small explorer class satellite, the Nuclear Spectroscopic Telescope Array (NuSTAR), which will image hard X-rays in the 8 to $80 \mathrm{keV}$ energy range.

We have already completed building the first two HEFT flight modules, which are shown in Figure 1. The angular resolution and effective area for HF1, which will be presented in Sections 4 and 5, exceed the HEFT requirements. This clearly demonstrates our ability to produce focusing hard X-ray optics with sufficient angular resolution and effective area to give meaningful astrophysics results. Through our parallel optics development program, we are demonstrating that we can also meet the requirements of future satellite missions. In order to achieve the science goals of HEFT while operating under the tight cost constraints of a balloon program, we have made a conscious decision to sacrifice our proven ability to achieve angular resolution significantly better than one arcminute for greater cost efficiency in building large effective area telescopes. In Section 6 we look toward the future and explain how we plan to improve future HEFT optics modules without adding significant extra cost. We also detail the steps required to transform the 45 " results we have achieved through prototype development work into full flight optics like those shown below in Figure 1.
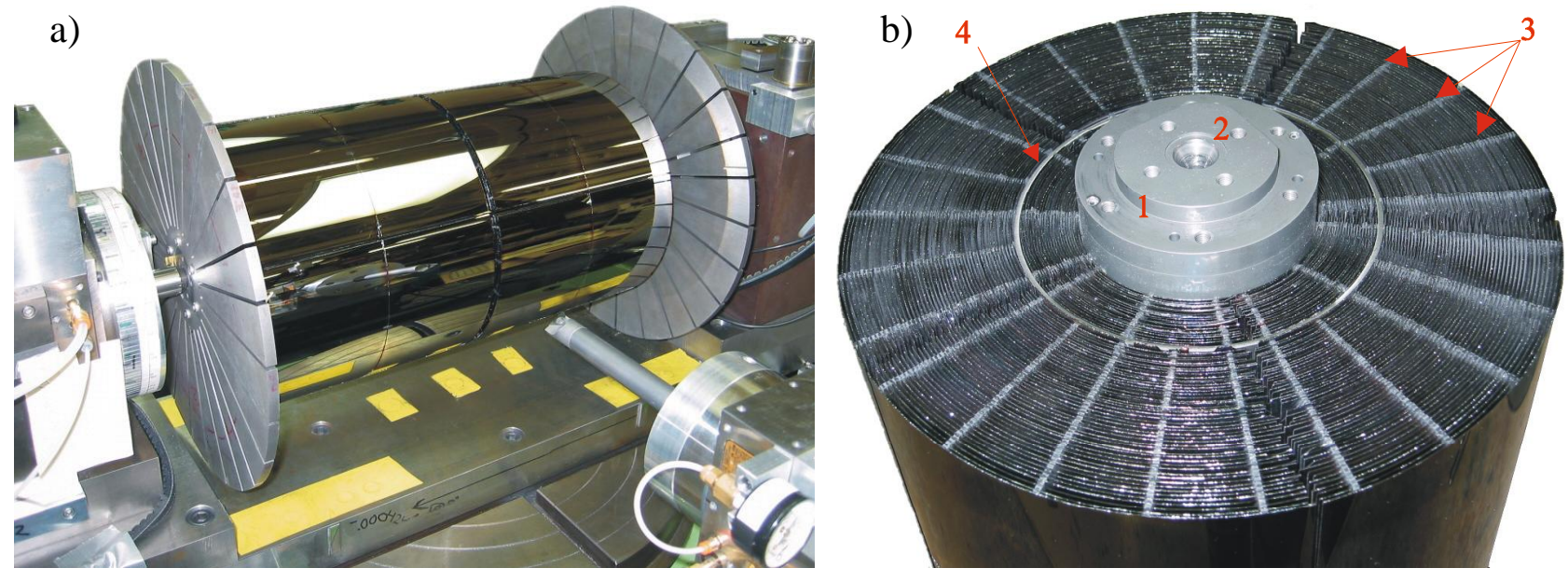

Figure 1: a) The second HEFT flight module (HF2) as it was being assembled at CPPI. b) An end view of the first Heft flight module (HF1) with 70 layers of segmented mirrors that each span $\sim 70$ degrees. The following details are noted that will be explained more fully in Section 3: 1 - the central titanium mandrel; 2 - precision alignment cone that defines the optical axis; 3 - stacks of graphite spacers; 4 - intermediate mandrel used for structural support when switching between 3 and 5 spacers per quint section.

\section{PRODUCTION OF HEFT MIRRORS}

A major accomplishment of the HEFT program has been the successful development of thermally-formed glass optics with performance exceeding the HEFT requirements. We begin with thin glass originally developed for flat panel displays that is smooth and flat on all relevant length scales. Our approach is to thermally form these micro-sheets using standard quartz mandrels and commercially available ovens. We begin by placing a glass micro-sheet on top of a concave mandrel inside of the oven. As the oven is heated to the appropriate forming temperature, the glass begins to form into the mandrel under the influence of gravity. Just before the glass touches the mandrel surface, the forming process is terminated by lowering the oven temperature. In this way, near net shaped optic substrates are produced without perturbing the excellent initial X-ray properties of the glass micro-sheet, even without the aid of highly polished and very expensive mandrels. 
The shells are characterized immediately after they are formed for quality control of the slumping process. An optical laser scanning apparatus, designed and built at Columbia's Nevis Laboratory and described more completely elsewhere, ${ }^{5}$ is used to characterize free standing cylindrical substrates. The laser can be arbitrarily moved over the cylindrical surface, and deviations in the reflected beam are measured by a two-dimensional position sensitive diode (PSD) to an accuracy of approximately 5". This apparatus can accommodate both coated and uncoated cylindrical optic pieces and the full range of sizes used for HEFT. In the case of uncoated glass substrates, the reflections from the front and backside of the glass are almost equal. Thus, the backside of the substrate is painted with a mixture of matte black watercolor and sugar. This provides for a low-cost and easily removable anti-reflection coating that reduces reflections from the back surface to approximately $4 \%$ of the combined reflection intensity. From axial scan measurements at multiple azimuth positions, the cylindrical surface can be reconstructed using software to remove shell alignment errors.

While the initial slumping parameters are be roughly determined from the raw glass properties, ${ }^{6}$ the slumping parameters for each oven must ultimately be tuned for each new production setup (e.g., new forming mandrel radius or different glass type - AF45 or D263). This initial tuning generally takes several days, but after that, the ovens settings are normally quite stable and subsequent substrates are produced with consistent angular performance. In this way, it is only necessary to perform laser metrology periodically for quality assurance, and only small fine tuning adjustments are required over weeks of mass production slumping with the same setup.

Currently, ten ovens are used for HEFT substrate production. Three to four substrates are normally produced every day in each oven. Once the correct slumping parameters are determined for a given production run, there is generally near $100 \%$ acceptance of the shells that are produced. Sample metrology on one piece from each oven is generally performed daily for quality assurance. If a sample is found to have poor performance, then the other pieces from that oven are also measured to determine if it is an isolated instance or if the oven slumping parameters must be retuned. After the pieces have been accepted, the original $20 \mathrm{~cm} \mathrm{x} 120$ degree pieces must be cut to the appropriate size $-10 \mathrm{~cm} \mathrm{x} \sim 70$ degrees using a scribe and break technique with better than $90 \%$ yield. The pieces are then packed and shipped to DSRI, where substrates are cleaning and coating at a rate of up to almost 100 pieces per day. ${ }^{7}$ With over two years of high throughput mirror production, we have refined our processes to be highly efficient. In this way, we are capable of producing two dozen mirrors with acceptable performance every day - enough for one telescope layer including four additional spares.

\section{ASSEMBLY OF HEFT OPTICS MODULES}

Our unique mounting process involves constraining glass segments to precisely machined graphite spacers that run along the optical axis. In this process, the nominally cylindrical glass segments are forced to a conical form, and in the process, radial mismatches and some small twists in the glass are removed. In order to achieve large effective area, concentric layers of glass are stacked on top of each other starting with a titanium mandrel. Graphite spacers are first epoxied to the mandrel and then precisely machined to the correct radius and angle. Next, a layer of glass and second layer of spacers are epoxied to the first set of spacers. These spacers are then machined to the appropriate radius and angle. This process is repeated until the requisite number of layers are assembled. A key point of this process is that each layer of spacers is machined with respect to the optic axis and not the last layer of glass. In this way, there is never any stack-up error during the telescope fabrication.

The assembly of two HEFT flight telescopes have been completed. For the first telescope, three spacers were used per quint section for the first 22 layers. At this point, a switch to five spacers per quint section was made. In order to make this change, an intermediate mandrel was required for structural support. The spacers on top of the $22^{\text {nd }}$ layer of mirrors were machined to give a tight fit with this intermediate mandrel (c.f., Figure 1b). The entire optic was removed from the assembly machine, and the intermediate mandrel was epoxied into place. The optic was remounted, and it was verified that the alignment end cones on the central mandrel indeed correctly repositioned the optic to better than a few arcseconds. The assembly process was continued using five spacers per quint section. During the time when the remaining 48 layers were added, the optic was removed from and replaced on the assembly machine two more times for prototype development work. Independent X-ray metrology performed on the optic after it was completed demonstrate that the optic can be removed and precisely aligned with no significant effect on the optic alignment (c.f., Section 4 for $\mathrm{X}$-ray results). The second HEFT flight module was completed in a similar fashion.

The monolithic structures produced by this method provide the extreme mechanical robustness that is necessary for the instrument to survive not only multiple balloon campaigns but also satellite deployment. The assembly apparatus has been designed using a deterministic manufacturing philosophy that places great importance on understanding the cause and effect of assembly errors. We have upgraded our mounting hardware and now position the spacers much more 
accurately, thus reducing the possible error contribution from spacer misalignment to a negligible level (<1”, c.f., Ref. 8 for discussion on spacer alignment errors).

A single technician was responsible for the assembly of HF2 at an average rate of four layers per normal work week. Our assembly technician is not only responsible for machining the spacers and mounting each layer of mirrors but is also responsible for performing periodic LVDT metrology and other quality assurance measurements in addition to receiving mirror shipments and keeping inventory. Our contingency plan to make up for any unforeseen schedule shortfall involves the aid of a second technician or student to make it possible to work through the entire week building a complete layer every day. In this way seven layers can be assembled each week.

More aggressive schedules can be accommodated by building multiple telescopes in parallel. The optic mandrel can be quickly and accurately aligned on the assembly machine, and it is also quick and easy to dismount and accurately remount an optic module. Indeed, we have demonstrated that there is no appreciable error in dismounting and remounting an optic while it is being assembled. Since the assembly machine is only required to machine the spacers, the optic mandrel can be removed from the machine after the spacers are machined, and the mirror segments can be assembled on a separate and much simpler apparatus. During the time necessary to assemble the glass segments on the first mandrel and allow the epoxy to cure, a second optic mandrel can be mounted on the assembly machine and its spacers can be machined. In this way, multiple optic units can be assembled in parallel using the same machine. Further, with a modest capital investment, the assembly machine can be duplicated to run multiple machines in parallel.

A great amount of flexibility in accommodating different sizes and types of substrate is also inherent in this method. The assembly apparatus design can be modified to accommodate an optic module with an arbitrarily large radius. We have already demonstrated the ability to use an intermediate mandrel. Concentric multi-mandrel designs can be used to reduce risk during the production process and improve production efficiency by dedicating separate machines to build different sections of the telescope. In this way, it is easy to envision scaling our mounting method to accommodate tight schedules for even the largest satellite missions.

\section{HF1 ANGULAR RESOLUTION}

The angular resolution of the first HEFT optic module (HF1) was characterized using several metrology techniques throughout the assembly process. These methods will first be explained, and then the performance results obtained from them will be compared.

\subsection{LVDT Metrology}

An important advantage to our mounting method is that metrology can be performed in situ as the optic is being assembled. An air-bearing Linear Variable Differential Transformer (LVDT) built by CPPI* was used to perform low force surface metrology on the back surface of the mounted mirrors. The thermally formed glass is very uniform in thickness as has been verified with our laser scanning apparatus, and thus, surface metrology performed on the back side of the glass is indicative of the front surface which serves to focus the X-rays. LVDT data are recorded for multiple axial scans at different azimuth positions to generate a complete surface. An example of the raw LVDT data is shown in Figure 2. While the LVDT device itself is linear over a 100 um range with an accuracy of $0.5 \%$, a significant amount of noise is recorded through our data acquisition system. Also, particles of dust ${ }^{\dagger}$ can also cause jumps in the LVDT data. In order to clean up the data, the surface derivatives are clipped to remove any discontinuities in the surface profile introduced by particulates on the substrate, and a low pass filter is applied to the data to remove noise introduced in the data acquisition process. The processed data is shown by the darker solid lines in Figure 1. This analysis procedure is quite effective and has been shown to compare well with X-ray data in past prototype development. ${ }^{8.9}$ A raytrace procedure is used to generate the two-bounce image off the upper/lower shell combination. Since the LVDT scans are stopped and started by hand, the scans are normally not uniformly performed over the entire $10 \mathrm{~cm}$ shell length. In order to efficiently analyze the data, all of the scans are truncated to the a common scan range where data for all scanned angles is available. In this way, the LVDT data is normally over $8.5-9.0 \mathrm{~cm}$.

\footnotetext{
* http://www.coloradoprecision.com/LVDT2.htm

$\dagger$ Our dust collection system efficiently removes the graphite dust generated during machining. While dust particles can on rare occasion cause spikes in the LVDT data as shown in Figure 2, dust does not significantly affect the telescope performance. The telescope throughput is normally extremely good ( 90\%), and the throughput losses are completely accounted for by geometric shadowing effects (c.f., Section 5).
} 


\section{2 $8 \mathrm{KeV}$ X-ray Performance Metrology at DSRI}

High resolution X-ray measurements at $8.048 \mathrm{keV}$ were performed at DSRI. A triple-axis diffractometer configuration utilized high-resolution, perfect channelcut monochromator and analyzer crystals (both $\mathrm{Si}(220)$ ) in a non-dispersive configuration. The optic was first aligned optically so that it rotates about its axis with no visible wobble in precisely aligned pinholes at each end of the optic module that define the optical axis. The Xray beam itself was then used to align the optic every $\sim 30$ degrees. This alignment was performed by rotating the optic in the horizontal plane and finding the maximum X-ray intensity through the pinholes at the front and back ends of the optic. In this way, residual wobble from the mechanical alignment of the optic was removed. It is important to emphasize that a Wolter-I optic is an imaging instrument. In this way, misalignment of the optic will not cause a displacement in the resulting image. The only consequence of any optic misalignment is that the measurements will be performed at a changing off-axis angle. The optic performance is constant up to several arcminutes off axis and only the throughput will be slightly degraded. The alignment of the optic is in fact estimated to be better than 15", and thus, it will have an insignificant effect on the resulting HPD measurements and the throughput will be degraded at most by a few percent.

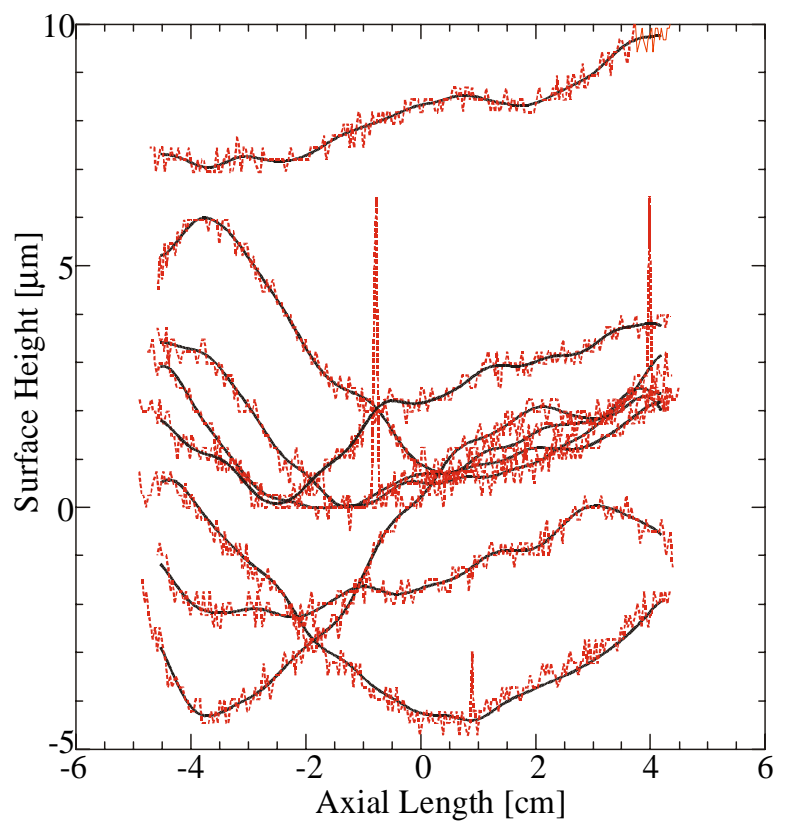

Figure 2: Segment 28d5 shown as an example of the LVDT analysis. The raw data is shown by the dotted lines. Surface slopes greater than 10' are first clipped to remove surface discontinuities. Then, a fifth-order Butterworth filter with a frequency cutoff of 1 cycle per $\mathrm{cm}$ has been applied to the raw data to remove noise introduced during data acquisition. The resulting filtered data is shown by the solid lines.

Scattering measurements are performed by translating the optic into the X-ray beam and subsequently rotating the analyzer crystal to probe the angle of the scattered radiation. In this way, the conic approximation error inherent in the optic design is not measured. The analyzer crystal accepts 5" as a nearly perfect step function. By scanning the analyzer crystal, a histogram of the reflected X-rays in angle space is recorded. Due to the excellent crystal resolution, essentially no background exists in this measurement and only small systematic uncertainties ( 5") are associated with co-adding the individual scans. This metrology method is thus very simple to analyze and provides a very accurate composite twobounce image of the upper and lower shells at multiple azimuth positions.

As with the LVDT metrology, the X-ray pencil beam scans are not always performed over the entire $20 \mathrm{~cm}$ length of the combined set of shells. A compromise between measuring the full shell length and changing the slit size for every layer was made. One slit size was generally used for $\sim 6$ layers. This is because the slit size of the collimator in front of the optic was not always set to the exact size of the shell: (Shell Length) $\times($ Angle $)=0.34-1.0 \mathrm{~mm}$. In this way, up to $10 \%$ of the outer portion of the shells can be missed. On average only $\sim 3 \%$ of the shell length is not measured.

\subsection{High Energy X-ray Metrology at ESRF}

The BM05 beamline at the ESRF synchrotron facility was used to perform high energy X-ray measurements. A double bounce $\mathrm{Si}(111)$ monochromator and beam collimators were used to generate an in plane divergence of 1" for the X-ray beam. The monochromator has energy range of 15 to $70 \mathrm{keV}$. The alignment of the optic was performed similarly to the DSRI setup, and as with the $8 \mathrm{keV}$ measurements, pencil beam scans were performed. However, a CCD detector was used at ESRF to actually generate a spatial image instead of using an analyzer crystal to measure the angular distribution of the focused X-rays. Due to space constraints, the CCD detector could not be placed at the focal point of the optic located $6000 \mathrm{~mm}$ from the optic center, but instead was positioned $2457 \mathrm{~mm}$ from the optic center. The only consequence of the shorter effective focal distance is an increase in the conic-approximation error folded into the image. For the inner mandrel layers (1-22), images were recorded over the full $20 \mathrm{~cm}$ length of the shell. This results in a 2.4 times increase in the conic approximation error. However, this error is still never more than 20" and has a small impact upon the performance of these shells. Measurements for all of the other layers were performed for the a-d and b-c 
combinations of $10 \mathrm{~cm}$ segment shells. In this way, the conic approximation error is only slightly (1.2 times) greater than the actual conic approx error.

Given a Gauss distribution of slope defects, the conic approximation contribution to the overall HPD can be approximated as

$$
\mathrm{HPD}_{\text {total }}^{2} \cong \mathrm{HPD}_{\text {axial }}^{2}+2 \mathrm{HPD}_{\text {conic }}^{2} \text {. }
$$

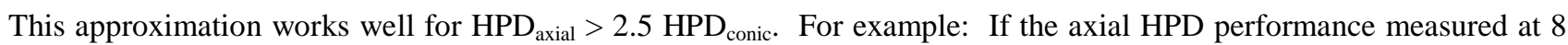
$\mathrm{keV}$ is 75 " for the last 10 layers where the conic approximation in the ESRF setup is $~ 20$ ", then it is expected that the conic approximation will add an additional $~ 5$ " to the total HPD measured at 18, 40, and $50 \mathrm{keV}$, and thus yielding an HPD of 80 ". The actual conic-approximation error for the HF1 telescope (6 m focal length) at these layers is $\sim 16 "$, which would add only $\sim 3$ " to the axial HPD. In this way, the true total HPD would be 78 ". In general all of the conicapproximation corrections will be less than 5" for HF1.

Unlike the $8 \mathrm{keV}$ measurements, a significant amount of background is measured using the ESRF setup. However, this background can be efficiently subtracted. While this background is normally quite flat as illustrated in Figure 3 for a scan on a layer 28 segment measured at $40 \mathrm{keV}$, it does begin to have structure once the total measurement throughput becomes low. However, a background subtraction procedure (explained more fully in Figure 3 ) has been developed to deal with this problem. The systematic uncertainty in these high-energy X-ray measurements is estimated to be $\sim 10 \%$.
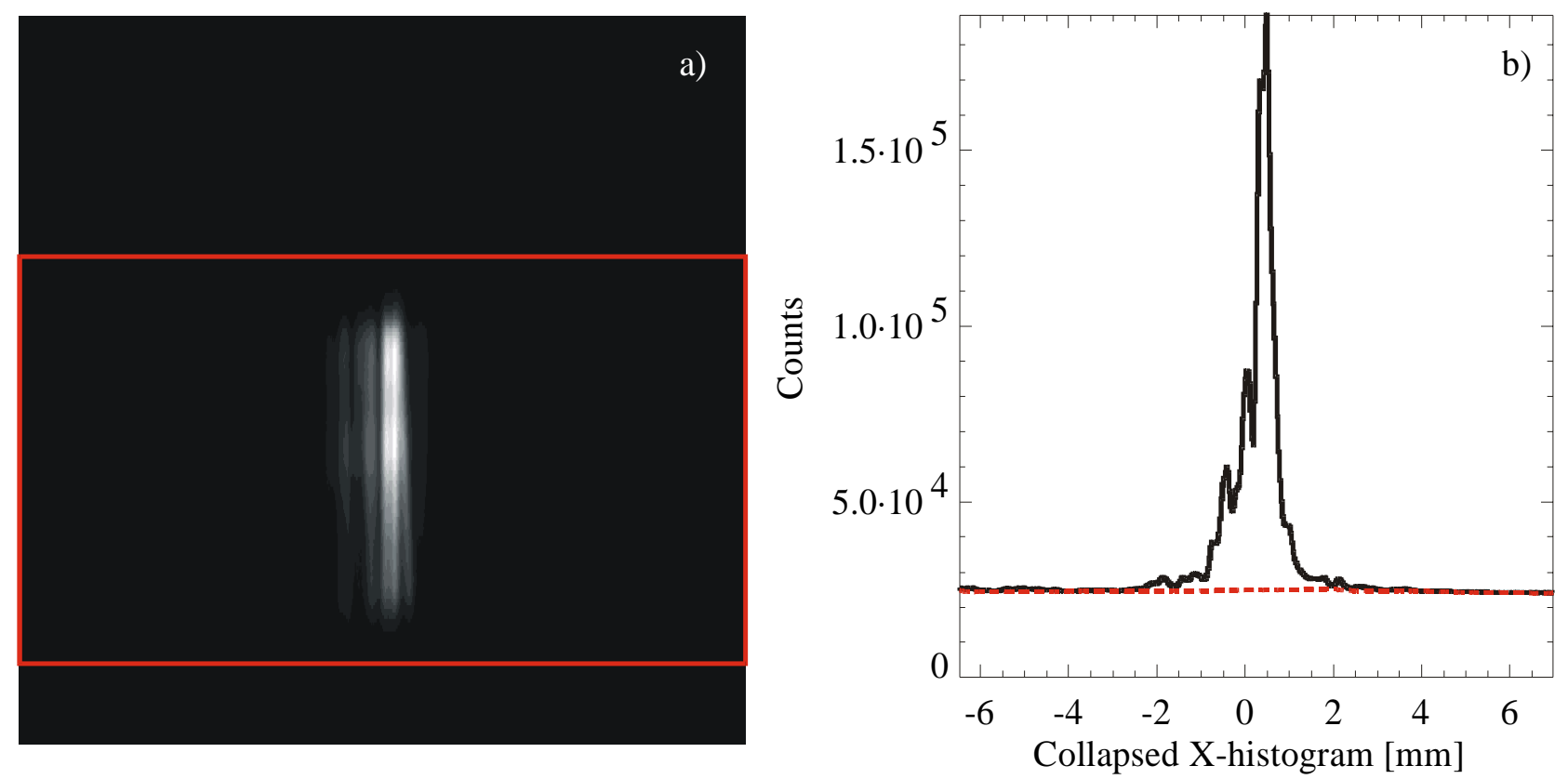

Figure 3: CCD image (7.5 um pixel size) at a single angle for Layer 28, Quint 5, a-d segment is shown in a). The vertical distribution is mainly due to the $1 \mathrm{~mm}$ beam size (a secondary effect arises from the out-of-phase roundness error). The horizontal distribution is due to the optic performance. The red boundaries illustrate the approximate cuts used for background subtraction. The data within the highlighted boundaries are collapsed to form the 1-d histogram shown as the solid line in b). The data outside the highlighted boundaries are collapsed and appropriately normalized to determine the nearly flat 1-D background histogram shown as the dashed line in $b$ ).

\subsection{Metrology Comparison}

About $60 \%$ of the telescope was measured at $8 \mathrm{keV}$; layers 27 and above at $18 \mathrm{keV}$; the entire telescope was measured at both 40 and $50 \mathrm{keV}$; only layers $10,16,20,28$, and 40 at $68 \mathrm{keV}$; and on average $\sim 1$ quint per layer with LVDT. The qualitative comparison of the image performance for the LVDT, $8 \mathrm{keV} \mathrm{X-ray,} \mathrm{and} \mathrm{high} \mathrm{energy} \mathrm{X-ray} \mathrm{data} \mathrm{is} \mathrm{generally}$ quite good. Figure 4 shows this comparison at 8 angles for Layer 28, Quint 5, one of the only quint segments for which all X-ray energies and the LVDT data are available. Only the events inside of the central 6' diameter were used in calculating the HPDs; any events outside this range are considered to be lost and not counted toward the effective area. This is a very reasonable and practical cutoff since the HPD becomes more sensitive to background subtraction for the 
high energy X-ray scans (note that no background subtraction was required for the $8 \mathrm{keV} \mathrm{X}$-ray data). While qualitatively the comparison looks quite good, there is quite a bit of variation in the HPDs determined at each angle. This variation is due to differences in the exact axial and azimuth extent over which each measurement was performed as well as systematic errors associated with the measurements.

Table 1 details the HPD performance for quint sections within the middle layers (25-48) that have both LVDT and X-ray data. The shell performance is expected to be independent of energy, and indeed, the measurements at different energies normally agree quite well with each other. The standard deviation from the mean in the five HPD measurements is typically $\sim 15 \%$ for these shells. The combined performance of all available data weighted by effective area (including throughput and multilayer reflectivity) is detailed in Table 7. The total performance of the entire telescope is $1.3 \pm 0.1^{\prime}$ at $40 \mathrm{keV}$ with the inner shells having $1.6 \pm 0.1$ ' performance, the middle shells $1.1 \pm 0.1$ ', and the outer shells $1.4 \pm 0.1$ '. The performance is of course dependant on energy due to the energy dependant weighting of the data.

Table 1: Comparison of LVDT and X-ray HPD results for middle layer group.

\begin{tabular}{|rrrrrr}
\hline Segment & LVDT & $\mathbf{8} \mathbf{~ k e V}$ & $\mathbf{1 8} \mathbf{~ k e V}$ & $\mathbf{4 0} \mathbf{~ k e V}$ & $\mathbf{5 0} \mathbf{~ k e V}$ \\
\hline HF1_2804 & $0.8^{\prime}$ & $0.9^{\prime}$ & $1.3^{\prime}$ & $1.2^{\prime}$ & $1.1^{\prime}$ \\
HF1_2805 & $1.0^{\prime}$ & $1.1^{\prime}$ & $0.8^{\prime}$ & $0.7^{\prime}$ & $0.9^{\prime}$ \\
HF1_2901 & $0.9^{\prime}$ & $1.0^{\prime}$ & $1.1^{\prime}$ & $1.2^{\prime}$ & $1.1^{\prime}$ \\
HF1_2902 & $1.0^{\prime}$ & $0.9^{\prime}$ & $0.8^{\prime}$ & $0.8^{\prime}$ & $0.9^{\prime}$ \\
HF1_2905 & $1.2^{\prime}$ & $1.1^{\prime}$ & $0.8^{\prime}$ & $1.0^{\prime}$ & $0.9^{\prime}$ \\
\hline HF1_3501 & $1.1^{\prime}$ & $1.1^{\prime}$ & $0.9^{\prime}$ & $1.8^{\prime}$ & $1.1^{\prime}$ \\
HF1_3504 & $1.0^{\prime}$ & $1.2^{\prime}$ & $1.3^{\prime}$ & $1.4^{\prime}$ & $1.4^{\prime}$ \\
HF1_3505 & $1.1^{\prime}$ & $1.4^{\prime}$ & $1.2^{\prime}$ & $1.3^{\prime}$ & $1.1^{\prime}$ \\
HF1_3602 & $0.9^{\prime}$ & $1.0^{\prime}$ & $0.9^{\prime}$ & $0.7^{\prime}$ & $1.0^{\prime}$ \\
HF1_3603 & $0.7^{\prime}$ & $0.9^{\prime}$ & $1.5^{\prime}$ & $1.3^{\prime}$ & $1.5^{\prime}$ \\
HF1_3604 & $1.2^{\prime}$ & $1.4^{\prime}$ & $1.2^{\prime}$ & $1.0^{\prime}$ & $1.2^{\prime}$ \\
HF1_3703 & $1.4^{\prime}$ & $1.7^{\prime}$ & $0.9^{\prime}$ & $1.0^{\prime}$ & $0.9^{\prime}$ \\
HF1_3704 & $1.2^{\prime}$ & $1.4^{\prime}$ & $1.1^{\prime}$ & $1.3^{\prime}$ & $1.1^{\prime}$ \\
\hline HF1_3804 & $0.9^{\prime}$ & $0.9^{\prime}$ & $1.1^{\prime}$ & $1.1^{\prime}$ & $1.1^{\prime}$ \\
HF1_3905 & $1.0^{\prime}$ & $1.1^{\prime}$ & $0.9^{\prime}$ & $0.7^{\prime}$ & $0.8^{\prime}$ \\
HF1_4001 & $0.6^{\prime}$ & $0.7^{\prime}$ & $0.9^{\prime}$ & $0.8^{\prime}$ & $0.9^{\prime}$ \\
HF1_4501 & $1.1^{\prime}$ & $1.1^{\prime}$ & $1.2^{\prime}$ & $1.6^{\prime}$ & $1.4^{\prime}$ \\
HF1_4602 & $1.4^{\prime}$ & $1.4^{\prime}$ & $1.3^{\prime}$ & $1.3^{\prime}$ & $1.5^{\prime}$ \\
HF1_4703 & $1.4^{\prime}$ & $1.4^{\prime}$ & $1.3^{\prime}$ & $1.3^{\prime}$ & $1.2^{\prime}$ \\
\hline HF1_4804 & $1.5^{\prime}$ & $1.7^{\prime}$ & $1.8^{\prime}$ & $2.0^{\prime}$ & $1.8^{\prime}$ \\
\hline Average & $\mathbf{1 . 0 7}^{\prime}$ & $\mathbf{1 . 1 7}$ & $\mathbf{1 . 1 1}^{\prime}$ & $\mathbf{1 . 1 8}^{\prime}$ & $\mathbf{1 . 1 5}^{\prime}$
\end{tabular}

Table 2: HPD performance comparison of LVDT and X-ray measurements for the inner (layers 1-22), middle (layers 2548), and outer (layers 49-72) portions of the HF1 telescope. The performance results for the entire telescope is also listed. These measurements are all weighted by the effective area (see Section 5 for effective area measurements). Thus, the results will be somewhat energy dependant, especially for the combined telescope. The weighted values for the middle layers are also different from the average values listed in Table 1.

\begin{tabular}{|l|r|rr|rr|}
\hline \multicolumn{1}{|c|}{ Group } & LVDT & 8kev & 18kev & 40kev & 50kev \\
\hline Inner & $1.7^{\prime}$ & $1.7^{\prime}$ & & $1.6^{\prime}$ & $1.7^{\prime}$ \\
\hline Middle & $1.1^{\prime}$ & $1.2^{\prime}$ & $1.1^{\prime}$ & $1.1^{\prime}$ & $1.1^{\prime}$ \\
Outer & $1.2^{\prime}$ & $1.3^{\prime}$ & $1.4^{\prime}$ & $1.4^{\prime}$ & $1.5^{\prime}$ \\
\hline \hline All & $1.3^{\prime}$ & $1.3^{\prime}$ & $1.2^{\prime}$ & $1.3^{\prime}$ & $1.5^{\prime}$
\end{tabular}

\section{HF1 EFFECTIVE AREA}

Due to the faint nature of most hard X-ray sources, the effective area is equally import to the angular resolution. A highly nested optic is required to achieve large effective area, part of which will inevitably be obscured by structural support. Past soft X-ray telescopes using segmented focusing optics have also reported significant losses stemming from geometric factors such as shadowing due to mirror misalignments, but these losses have not always been completely understood.

In the case of HEFT and other similar hard X-ray optics, shadowing becomes especially important due to the smaller graze angles required for hard X-rays. Further, two $10 \mathrm{~cm}$ segments are used in HEFT to form each of the $20 \mathrm{~cm}$ upper and lower mirror layers. In this way, there are actually three shell intersections where shadowing can occur instead of just one. The HEFT assembly approach is particularly adept at minimizing such shadowing because each mirror segment is constrained by several spacers machined to the correct radius with approximately 1 um accuracy. Since each layer is machined with respect to the optic axis, an error in any single mirror will not affect the next - there is never any stack-up error. 

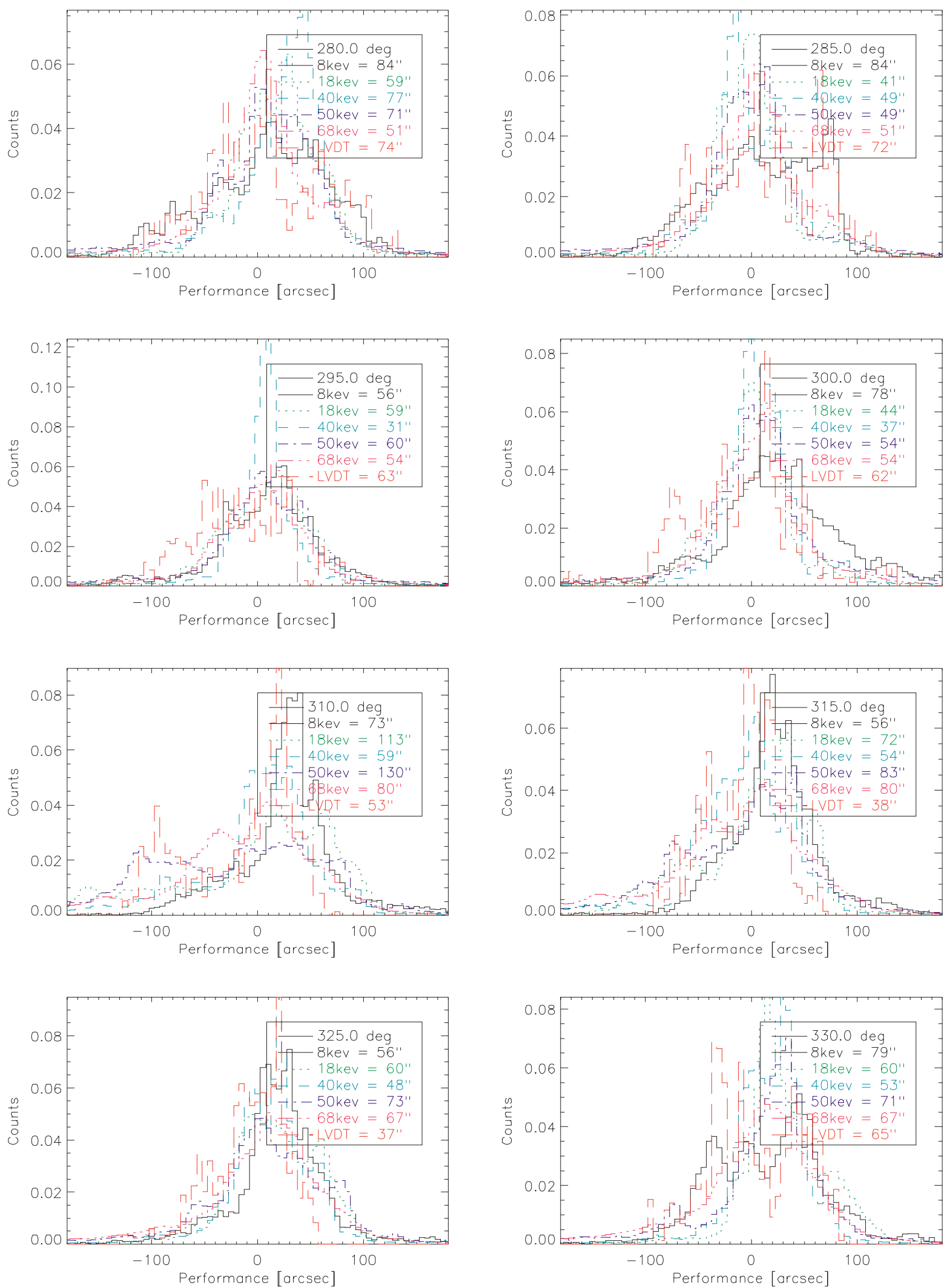

Figure 4: Comparison of LVDT and X-ray data for Layer 28, Quint 5 
The thermally formed substrates used for HEFT are not perfectly formed to the correct conic geometry. In fact, they are only nominally cylindrical, and substrates formed using the same slumping mandrel are used on several different radii in order to reduce mandrel costs. Thus, there will always be an in-phase roundness error associated with these mirrors as they buckle inward and outward between spacers due to radial mismatch. The radius error for mirrors mounted on HF1 typically reached a maximum of $\pm 10-15$ um between spacers for the outer layers that used five spacers per quint section, but was often a little higher for the inner layers that used only three spacers per quint section. There is a gap of $8 \mathrm{~mm}$ between the upper and lower layers in the HEFT design which allows for an extra $\sim 30$ to 80 um in tolerance for the mirror radius of the inner to outer layers, respectively. Thus, only the intersection of the two $10 \mathrm{~cm}$ pieces that form each upper and lower quint section will normally cause any significant shadowing. Losses from shadowing in this manner will be considered to be loss in axial throughput - in contrast to losses from structural obscuration that will be dealt with later.

In addition to assessing the angular performance, the LVDT data was used to determine the axial throughput using raytrace calculations. The result of these throughput calculations are shown in Figure 5a. We have also determined the axial throughput at DSRI using $8 \mathrm{keV}$ scattering measurements in a double-axis diffractometer configuration without the analyzer crystal in place. For these measurements, the $8 \mathrm{keV}$ X-ray flux was measured every 2.5 degrees with a pin diode detector and calibrated with the direct beam similarly to the performance measurements. As with the $8 \mathrm{keV}$ performance measurements, there is very little background involved in these measurements. In addition, the normalization of the throughput measurements is much better than the performance measurements, and they are much quicker to perform since scanning through angle space is not necessary. At $8 \mathrm{keV}$, the combined reflectivity of the upper and lower layers ranges from $95 \%$ for the innermost layer to $79 \%$ for the outermost layer. Further, the reflectivity at 8 $\mathrm{keV}$ is not as strongly dependent upon the exact multilayer coating including the micro-roughness as it is at higher energies. Thus, the X-ray measurements provide a good method of directly assessing how much the effective area is degraded due to obscuration and other geometric effects. The axial throughput results extracted from this $8 \mathrm{keV}$ data are also plotted in Figure 5a along with an analytic approximation to the $8 \mathrm{keV}$ and LVDT.

For the first 22 inner mandrel layers where only three spacers were used for each mirror segment, the axial throughput becomes increasingly degraded as the conic angle decreases toward the innermost layer. This trend is expected because a given roundness error will cause a relatively longer shadow for shallower graze angles than the same error will cause for larger graze angles. After the switch to five spacers starting at layer 25, the axial throughput was consistently $~ 90 \%$. Because of the good agreement between the LVDT simulation and the $8 \mathrm{keV} \mathrm{X-ray} \mathrm{illumination} \mathrm{measurements,} \mathrm{we} \mathrm{can}$ be confident that the loss in axial throughput is completely accounted for by geometric shadowing effects. Any degradation in throughput due to other factors such as scattering from dust particles or imperfections in the multilayer coatings must be minimal, as is indeed expected.

Each HEFT optic module will be mounted on the gondola using a support structure that will cover the gaps between quint sections. The five supports will each be $\mathrm{w}_{\text {gap }}=3 \mathrm{~mm}$ wide - about the same width as the gap between quint sections. While the spacers themselves are only $1.6 \mathrm{~mm}$ wide, a small amount of epoxy excess around the spacer will cause added obscuration for each spacer. On average, each spacer obscures $\mathrm{w}_{\text {spacer }} \cong 2.5 \mathrm{~mm}$ of the segment. Thus, the total obscuration will be

$$
\varepsilon_{\text {obscuration }}=\left(\mathrm{n}_{\text {spacers }} \mathrm{w}_{\text {spacer }}-\mathrm{n}_{\text {segments }} \mathrm{w}_{\text {gap }}\right) /\left(2 \pi \mathrm{r}_{\mathrm{uo}}\right) \text {, }
$$

where $n_{\text {spacers }}$ is the number of spacers, $n_{\text {segments }}=5$ is the number of mirror segments, and $r_{\text {uо }}$ is the middle radius of the upper layer. The total obscuration for the first two HEFT modules will range from 10-20\%.

The high energy performance measurements detailed in Section 4 could in principle have been used to determine the energy dependence of the effective area. Unfortunately, there were severe problems with the overall calibration of these measurements that made it impossible to extract an accurate normalization. However, separate energy scan measurements from 18 to $70 \mathrm{keV}$ were also performed at ESRF on a number of individual quint sections. The normalization of these energy scan measurements is better understood. Results of these measurements, including a more general study of the W/Si multilayers for HEFT, are detailed in Madsen et. al. ${ }^{2}$ Taking into consideration the throughput measured at $8 \mathrm{keV}$, these shells were shown to have an energy response consistent with theoretical predictions based on the coating recipes.

Using the theoretical multilayer reflectivities, the structural obscuration from Equation 2, and the measured axial throughput, the effective area was calculated for both on- and off-axis sources. The on-axis effective area is plotted 
logarithmically versus layer for several energies in Figure 5b. The total effective area for HF1 is plotted as a function of energy in Figure 6 for both on- and off-axis sources.
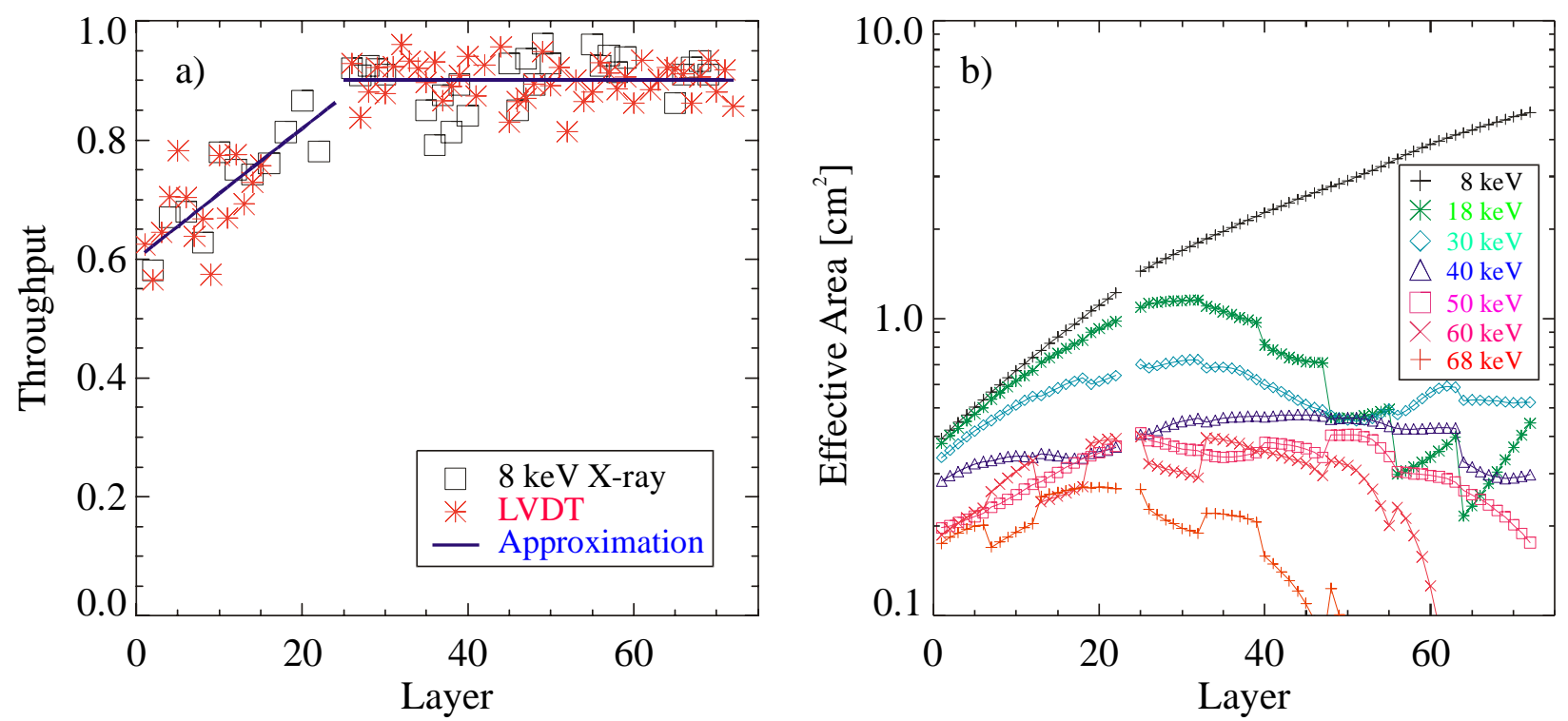

Figure 5: a) HF1 throughput determined from raytrace calculations using LVDT data and measured directly using 8 keV X-rays. The $8 \mathrm{keV}$ measurements are the average for the entire layer as are the LVDT measurements for the inner layers up to layer 16 . The rest of the LVDT measurements are typically only for one sample quint segment. The errors in each of these measurements are estimated to be less than 5\%. b) The effective area is plotted logarithmically versus layer for several energies. This effective area includes the theoretical multilayer reflectivities, structural obscuration and axial throughput.

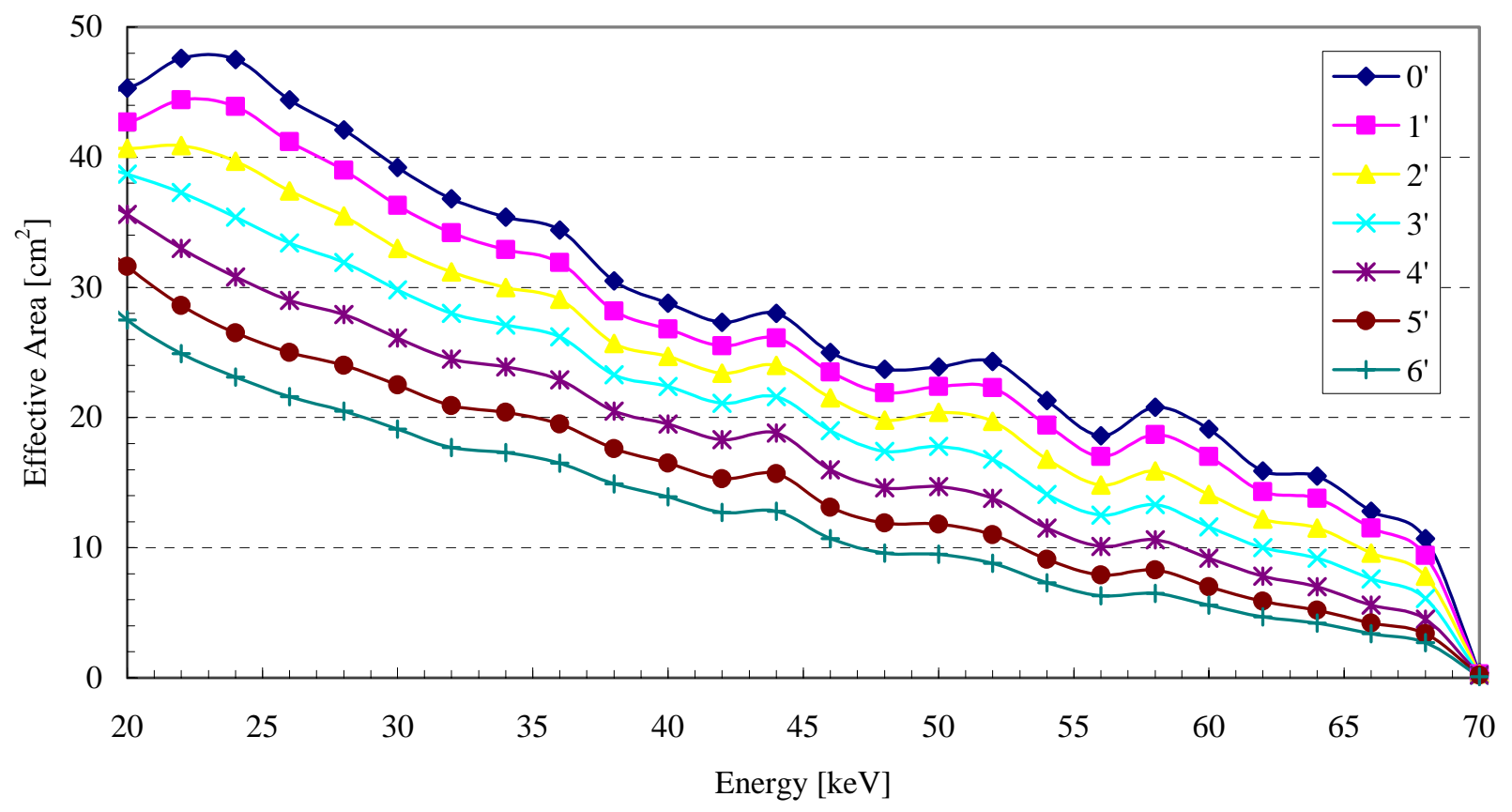

Figure 6: The total effective area response for HF1 is plotted versus energy for both on-axis sources (0') and sources 1' to 6' off-axis. 


\section{OUTLOOK FOR HARD X-RAY OPTICS DEVELOPMENT}

Through HEFT we are demonstrating key technologies that are proposed for future satellite missions. However, HEFT is not simply a prototype for future telescopes. Indeed, HEFT will be among the first focusing telescopes to yield significant hard X-ray science returns as a balloon mission. In building HEFT optics, our goal has been to achieve the highest sensitivity possible under a cost constrained budget. In this way, several key compromises have been consciously made - most notably we have sacrificed some angular resolution in order to more efficiently build multiple telescope modules. In contrast to satellite missions where space and weight are at a premium, the HEFT gondola is capable of carrying up to fourteen optic modules. Thus, we have concentrated on achieving high sensitivity though large effective area rather than extremely high resolution mirrors.

Parallel to our efforts to produce flight grade telescopes for HEFT, we have an active research and development program to improve the angular resolution of our segmented glass optics. Key to this effort is performing detailed metrology of the glass substrates before they are mounted. It has been and will continue to be impractical to perform and analyze detailed metrology on every substrate produced for the HEFT balloon born mission. In spite of this, our method of sampling for quality assurance has proven to be quite effective, and we have achieved our one arcminute goal for the central and most important portion of the first HEFT optic module, HF1. Indeed, the performance of the entire optic is $1.3^{\prime}$ at $40 \mathrm{keV}$.

By performing full metrology and implementing a more stringent acceptance criteria on the substrate performance, we have demonstrated 45 " resolution in a prototype optic. ${ }^{10}$ The substrates chosen for this prototype were from the same ensemble used to build the HEFT flight telescopes. The difference is that with this prototype, we were more effectively able to reject poorer performing shells that would have otherwise degraded the overall performance. Ultimately, $50 \%$ of the substrates available for this 45 " prototype were rejected based on the detailed laser metrology of every substrate. This is in contrast to HEFT where sample metrology is performed only periodically for quality assurance of the general slumping parameter settings and nearly every substrate is actually accepted - most with no knowledge of the actual freestanding performance.

It should be reemphasized that the axial figure is not greatly changed in our mounting approach, and in building this 45 " prototype from the same ensemble of substrates used to produce $\sim 60$ " shells in HF1, we have demonstrated that we are quite effective in predicting the axial figure of the mounted shells from freestanding laser data and only accepting the best shells. The key to our mounting approach is that we force nominally cylindrical shells to the correct radius, and in the process remove much of the twists in the shells that lead to an out-of-phase roundness error. This residual roundness error is what we have a much more difficult time predicting from the laser data. In this way, we recognize a limitation in our current method of rationally 'cherry picking' the best shells - even in building this 45" prototype through down selecting shells. Indeed, if the phase errors (i.e., residual twists) in the LVDT data of these shells are removed, the performance of these shells become $23 \%$ better. This indicates that we could achieve as good as 35 " performance with these same shells if we were able to 'perfectly' constrain their overall shape. In order to better evaluate the effect the mounting process will have on the phase errors of the glass substrates, we have designed a cylindrical mount fixture that serves as a surrogate for the mounting process at CPPI. This surrogate mount has precision machined spacers against which substrates can be mounted to simulate the full fabrication process in a non-destructive manner (i.e., without epoxy). Studies in this way have already have already yielded valuable insight into the mounting process.

Another way to achieve better performance is by increasing the number of spacers per mirror segment. There was a huge jump in the HF1 performance from 90" for layers $20-22$ to $\sim 60$ " for layer $26-28$. This was largely due the change from three spacers to five spacers. In addition to improved performance through a decrease in the out-of-phase roundness error, more spacers will also help reduce the in-phase roundness error (i.e., the buckle inward and outward between spacers) that can potentially degrade the throughput. For the inner layers that use only three spacers, this could significantly improve the throughput that currently reaches as low as $60 \%$ for the innermost layer. Each additional spacer will of course obscure more of the effective area. Thus, we plan to decrease the width of the spacers from their current width of $1.6 \mathrm{~mm}$ to $<1.0 \mathrm{~mm}$. The original choice of $1.6 \mathrm{~mm}$ wide spacers was based on a very conservative engineering approach, and we have calculated that thinner spacers will still provide sufficient support to maintain the structural integrity of the optic.

Each additional spacer requires extra time to machine. For the third HEFT telescope (HF3), we plan to begin with five spacers per quint section instead of three spacers as was the case for HF1 and HF2. The expected increased angular 
resolution and throughput will be well worth the extra assembly time and cost. Additional spacers are not as likely to be cost and time effective for HEFT, but would likely be incorporated into the design for satellite telescopes.

The outlook for our approach to building hard X-ray optics is very promising. We have demonstrated that we can efficiently build large effective area telescopes, and we have shown that we understand the response of these telescopes though the metrology presented in this paper. The steps to move from balloon grade optics to optics appropriate for satellite missions are clear. We simply require the high quality control of our current substrates that we have demonstrated through a 45 " prototype. Since we are limited by the substrate performance and our mounting method contributes negligibly to the overall performance, a change to even higher performing substrates mounted with our assembly approach promises to achieve even the most stringent angular resolution requirements of the most demanding satellite telescopes.

\section{ACKNOWLEDGEMENTS}

This technical progress described in this paper could not have been made without the expert and tireless work of our technical staff. Special Thanks to the staff at ESRF for their assistance with the high energy measurements. This work is supported by a NASA grant to Columbia University: NAG5-5260 (Thermally Formed Optics for X-Ray and GammaRay Astronomy), and by NASA grants to the California Institute of Technology with sub-awards to Columbia: Caltech No. 1019776 (High Energy Focusing Telescope) and Caltech No. 1046806 (Critical Technologies for the Constellation Hard X-Ray Telescope).

\section{REFERENCES}

${ }^{1}$ C. M. H. Chen, W. R. Cook, F. A. Harrison, J. Y. Lin, P. H. Mao and S. M. Schindler, "Characterization of the HEFT CdZnTe pixel detectors", Proc. SPIE, 5198-02, 2003.

2 K. K. Madsen, F. E. Christensen, C. P. Jensen, "X-ray study of W/Si multilayers for the HEFT hard X-ray telescopes", Proc. SPIE 5168-08, 2003.

${ }^{3}$ D. L. Windt, S. Donguy, C. Hailey, J. Koglin, V. Honkimaki, E. Ziegler, F. Christensen, F. Harrison, "Optical constants for hard X-ray multilayers over the energy range E = $35-180 \mathrm{keV}$ ”, Proc. SPIE 5168-06, 2003.

${ }^{4}$ K. S. Gunderson, F. E. Christensen, W. W. Craig, T. A. Decker, C. J. Hailey, F. A. Harrison, R. McLean, R. E. Wurtz, K. Ziock, "Laboratory performance of the high-energy focusing telescope's attitude control system", Proc. SPIE 516512, 2003.

5 M. Jimenez-Garate, W. Craig, C. Hailey, F. Christensen, and A. Hussain Opt. Eng. 39(11), p. 2982, 2000.

${ }^{6}$ M. Jimenez-Garate, Ph.D. thesis, Columbia Ph.D. (physics), 2001.

7 M C. P. Jensen, H. Chen, F. E. C. K. Madsen, and E. Ziegler, "Coating of the heft telescope mirrors: method and results", Proc. SPIE 4851(79), 2002.

8 J. Koglin, F. Christensen, J. Chonko, W. Craig, T. Decker, C. Hailey, F. Harrison, C. Jensen, M. Sileo, D. Windt and H. Yu, " Development and Production of Hard X-ray Multilayer Optics for HEFT," Proc. SPIE 4851, 67, 2002.

9 J.E. Koglin, H.C. Chen, F.E. Christensen, J. Chonko, W.W. Craig, T.R. Decker, M.A.Jimenez-Garate, C.J. Hailey, F.A. Harrison, C.P. Jensen, M. Sileo, D.L. Windt and H. Yu, "Development of Precision Hard X-ray Multilayer Optics with Sub-Arcminute Performance," Proc. SPIE 4851, 673, 2002.

${ }^{10}$ C. J. Hailey, F. E. Christensen, W. W. Craig, F. A. Harrison, J. E. Koglin, R. Petre, W. W. Zhang, "Fabrication and Performance of Constellation-X Hard X-ray Telescope Prototype Optics Using Segmented Glass”, Proc. SPIE 5168-11, 2003. 